\title{
Oligonucleotide bioanalysis: sensitivity versus specificity
}

"Coupling MSIMS with UPLC may improve both assay sensitivity and specificity by providing better chromatographic resolution and sharper peaks ... However, until now, challenges have existed for routinely analyzing oligonucleotides from biological matrices primarily due to the short life of UPLC columns."

Keywords: bioanalysis $\approx$ hybridization-ELISA $\approx$ LC-MS/MS $\approx$ oligonucleotide $\approx$ siRNA $\approx$ UPLC

The bioanalysis of oligonucleotide therapeutics is constantly evolving, primarily driven by drug development needs and regulatory requirements. The technologies have been comprehensively reviewed by Tremblay and Oldfield [1]. Each method has been used for different study purposes for a period of time. To support preclinical and clinical studies, assay robustness/ruggedness, sensitivity and specificity are critical features for obtaining reliable data from precious samples in a timely manner. With recent advances in analytical technologies, currently the three major technologies supporting the bioanalysis of oligonucleotide therapeutics, especially in good laboratory practice (GLP) environments, are hybridization-ELISA, LC-MS/MS and UPLC-UV (photodiode array).

\section{Hybridization-ELISA: less specific but sensitive for large oligonucleotides, no metabolite information}

Generally speaking, hybridization-ELISA (also known as hybridization immunoassay) provides the best assay sensitivity and throughput compared with other methods. Hybridization assays usually involve the hybridization of the target oligonucleotide to a capture probe (immobilization) and/or to a detection probe (signaling). Therefore, the assay requires little or no sample clean-up. But the assay has a narrow calibration range (usually 20-50-fold) and its robustness relies heavily on the quality of the reagents. The sensitivity and specificity of the assay depend on the choice of the methods/reagents and the structures of the target oligonucleotides.

For a 'sandwich hybridization' assay, the LLOQ can be as low as $100 \mathrm{pg} / \mathrm{ml}$ in plasma [2]. However, the target oligonucleotide needs to be long enough (usually $>20$-mer) to allow both the capture probe and the detection probe to bind stably to different parts of the molecule. This method is usually unable to distinguish the large metabolites (truncated n-1 and n-2 metabolites) from the full-length target oligonucleotide.

The 'hybridization-ligation' assay is much more specific and has comparable sensitivity [3]. The length requirement of the target is also much shorter, as the target oligonucleotide binds to part of the capture probe (also known as the template probe). The capture probe has a generic $5^{\prime}$-end overhang, which is complementary to a 5'-phosphorylated ligation probe. As the enzyme ligation efficiency is very low in the presence of a gap, the 3 -end truncated metabolites are difficult to detect using the ligation-based assay. The 5 -end metabolites, however, will interfere with the detection of the full-length oligonucleotide.

In theory, the 'nuclease-based hybridization' assay is more specific than the 'hybridizationligation' assay. It uses the properties of a single-strand-specific nuclease (S1 nuclease) to degrade the free capture probe and the non-fully matched hybrids [101]. Therefore, only the full length target oligonucleotides are expected to be detected. However, in practice, the sequence context and the intrinsic characteristics of the S1 nuclease commonly used with the digestion assay may also allow the detection of $n-1$ truncated metabolites [4].

The 'competitive hybridization' assay is an option if the sandwich or ligation assays are not feasible, due to the length or structure limit of the analyte oligonucleotide [5]. In addition to the low assay specificity, the reported limit of the quantification of a competitive hybridization assay is $900 \mathrm{pM}(\sim 5 \mathrm{ng} / \mathrm{ml})$ in plasma for a 15-mer phosphodiester oligonucleotide [6].

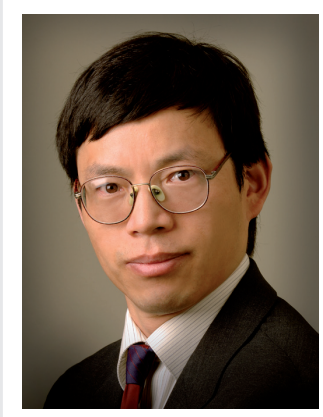

\section{Laixin Wang}

Tandem Labs, a LabCorp company, 112I East 3900 South, Suite C-105, Salt Lake City, UT 84I24, USA

Tel.: + I 80I 2932397

Fax: + 8013136495

E-mail: Laixin.Wang@Labcorp.com 
LC-MS/MS: specific \& sensitive for small oligonucleotides

LC-MS/MS has emerged as a powerful tool to quantify a variety of oligonucleotide therapeutics in biological matrices [6]. Contrary to hybridization-ELISA assays, LC-MS/MS is especially advantageous for quantifying relatively small oligonucleotide therapeutics (25-mer or shorter [7]. With three levels of discrimination (chromatography separation, molecular weight identification and product ion characterization) LC-MS/MS is one of the most specific bioanalytical methods for oligonucleotide therapeutics. Generally speaking, the sensitivity of an LC-MS/MS assay decreases when the size of an oligonucleotide increases due to the limit of LC resolution and the additional number of molecular charge states in the MS source [7]. Depending on the size of the target oligonucleotides, a 5-10 $\mathrm{ng} / \mathrm{ml}$ LLOQ has been achieved from 100- $\mu$ l plasma samples [8,9]. Compared with hybridization-ELISA assays, LC-MS/MS methods have a much wider dynamic range (up to three orders of magnitude) and no special reagents are required. It can also quantify intact double-stranded oligonucleotides, such as siRNA, by measuring either sense- or antisense-strand RNA. Many LC-MS/MS methods have been developed and validated to support preclinical and clinical studies of both single-stranded DNA oligonucleotides and double-stranded siRNAs [7].

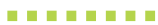

"Contrary to hybridization-ELISA assays, LC-MSIMS is especially advantageous for quantifying relatively small oligonucleotide therapeutics."

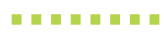

In addition to accurate and specific quantitations of the full-length oligonucleotides, the metabolites can also be analyzed simultaneously using the same LC-MS/MS assay if the standard reference materials are available [8]. In the meantime, the same LC-MS/MS platform can be used to analyze delivery vehicles, such as lipids or polymers in the study samples. Therefore, if sensitivity is adequate, LC-MS/MS is poised to become the first choice for the bioanalysis of oligonucleotide therapeutics.

LC-MS/MS has become a standard technique for the determination of small-molecule drugs in biological fluids for more than a decade. However, until recently, it has been less successful for the quantitative bioanalysis of therapeutic oligonucleotides due to the many challenges associated with their unique physicochemical and biological properties [10]. These challenges include:

- Multiple charge states and cation adducts in the ion source;

- Nonspecific binding to the components in biological matrices;

- Adsorption to LC column and injector systems;

- Chemical and enzymatic instability during sample storage and sample processing;

- Nonspecific binding to container surfaces.

Overcoming these challenges has been the key for the successful development and validation of an LC-MS/MS assay. As oligonucleotides are large polyanionic molecules they tend to bind matrix components, especially cationic components, which significantly affects the LC separation and MS ionization of the analyte oligonucleotides. Therefore, unlike the hybridization-ELISA assays, the biological samples need to be prepared and extracted appropriately prior to being analyzed via LC-MS/MS. Liquid-liquid extraction (LLE) with phenol/chloroform followed by SPE has been reported with recoveries greater than $70 \%$ for single-stranded DNA oligonucleotides [8]. A single step phenol/chloroform LLE extraction was found to be able to provide even better and more consistent recoveries from plasma and urine samples for all tested single-stranded oligonucleotides [9]. The key is to add the appropriate amount of ammonium hydroxide or other disrupting reagents to the sample to break down the oligonucleotide/ matrix binding complexes prior to proceeding with phenol/chloroform extraction. In addition, it is important to keep some competitive component(s), such as EDTA, in the extracts to reduce the nonspecific binding of the oligonucleotide to the LC system and, therefore, improve the peak shapes and prolong the column lifetime. For the same reasons, the singlestep LLE extracts can usually be stored longer (up to one week at $1-8^{\circ} \mathrm{C}$ ) in plastic plates/vials than corresponding extracts produced with the LLE-SPE combination method. However, the single-step LLE extraction yields very low recovery $(<20 \%)$ for double-strand siRNA. One possibility is that the LLE extraction conditions break down the hybridized siRNA duplex and the resulting single-strand RNA is rapidly degraded by the RNases in the samples. 
Fortunately, the Phenomenex ${ }^{\circledR}$ Clarity $^{\circledR}$-OXT SPE plates were proved to be effective for a onestep SPE extraction of the intact duplex siRNA oligonucleotides from plasma and urine. The extraction procedure needs to be slightly modified from the manufacturer-supplied protocol according to the structures of different siRNAs. To prevent the stickiness loss and the degradation of siRNA, RNArecover ${ }^{\mathrm{TM}}$ solution (a reagent containing RNase inhibitors and nonspecific binding inhibitors) must be added into the final extracts.

Another commonly encountered problem when working with oligonucleotide LC-MS/MS assays is that oligonucleotide concentrations gradually decrease with long-term storage in neat solutions when the concentrations are low. Most of the time, the loss of the oligonucleotides at low concentrations is caused by adsorption to the container's surface rather than by compound instability, since the sample losses are ameliorated when the samples are stored in glass rather than plastic containers. The addition of $1 \%$ of bovine serum albumin to the neat solutions also slows down the loss of samples. The same holds true for urine samples; long-term matrix stability in urine may fail at low concentrations for some of the oligonucleotide samples, however, successful long-term stability data is able to be generated if samples are treated with an appropriate amount of plasma immediately after urine collection. Adsorption is generally not an issue for plasma samples, but EDTA is highly recommended as an anticoagulant, since not only does EDTA stabilize the oligonucleotides by chelating the divalent metal ions, which are the cofactors of most DNA and RNA enzymes, but it can also improve the extraction recovery, presumably by competitive bindings.

\section{UPLC-UV: specific but not sensitive, providing metabolite information without standard reference materials}

UPLC has been extensively employed to reduce assay cycle time and improve assay sensitivity over conventional HPLC assays during the last 5 years [11]. Unlike LC-MS/MS methods that have three levels of identification, the discrimination of an intact oligonucleotide from its truncated metabolites solely relies on the chromatographic separation primarily based on the differences of their molecule sizes/lengths. Fortunately, the UV detectors are compatible with nonvolatile salts in the mobile phases. Therefore very strong ion-pair reagents, such as tetrabutylammonium sulfate and tributylammonium acetate, can be used as modifiers in mobile phases for ion-pair reverse-phase UPLC. As such, a full-length oligonucleotide (30-mer or shorter) can be readily separated from its $n-1$ and other truncated metabolites within $10 \mathrm{~min}$ on a C18 UPLC column, which makes the LC-UV assay reasonably fast to support the bioanalysis of oligonucleotides for studies with a large number of samples. For sample extractions, all of the above mentioned methods are usually suitable for UPLC-UV analysis. The typical LLOQ of a validated UPLC-UV assay is approximately $0.2-1.0 \mu \mathrm{g} / \mathrm{ml}$ [12]. The biggest advantage for a UPLC-UV assay is that the relative concentrations of all truncated metabolites can be estimated simultaneously even without standard reference materials.

\section{Perspective}

The bioanalysis of oligonucleotides is evolving along with the increased diversity of the oligonucleotide structure and the advancement of sample preparation/extraction and analytical technologies. Since oligonucleotide therapeutics are still relatively new biopharmaceuticals, the choice of bioanalytical methods is still based on the fit-for-purpose principle and should be evaluated on a case-by-case basis.

Hybridization-ELISA assays usually provide the best sensitivity for oligonucleotides with 16 nucleotides or longer, but the specificity and reproducibility are not as good as LC-MS/MS methods. Tremblay et al. recently reported a 'dual ligation hybridization' assay with improved specificity [13]. The dual ligation assay relies on a bi-enzymatic reaction comprising a commercially available DNA ligase and a polynucleotide kinase, to covalently connect two probe oligonucleotides ( $3^{\prime}$ - and 5'-probe) onto the analyte oligonucleotide with the help of a template oligonucleotide. The assay minimized the 3 '-end metabolite interference to less than $4 \%$ and the $5^{\prime}$-end metabolite interference to an undetectable level. The reported calibration curve range is $0.12-7.5 \mathrm{nM}(\sim 1-50 \mathrm{ng} / \mathrm{ml})$ in plasma for a 21-mer phosphorothioate oligonucleotide, which is comparable to the conventional hybridization ligation assay [3]. The author also combined the same dual ligation principle with quantitative PCR (qPCR) to quantify the same oligonucleotide [13]. Similar assay specificity was obtained, and a 75-2000 nM calibration curve range was achieved by analyzing dried blood spot samples [13]. However, the dual ligation 
qPCR assay used seven primer/template/probe oligonucleotides and three enzymes to quantify one analyte oligonucleotide. It will be challenging to use such a complex assay for the routine bioanalysis of oligonucleotides in a GLP environment.

LC-MS/MS assays do not require any special reagents. Triple-quadrupole MS has been the workhorse for the quantification of drugs in bioanalytical industries. Using full-scan mode (Q1 scan) on a Sciex API5000 ${ }^{\mathrm{TM}}$, an oligonucleotide always produces multiple $\mathrm{m} / \mathrm{z}$ peaks corresponding to the molecule bearing different amounts of negative charges. Therefore, the sensitivity decreases when the length/size of the oligonucleotide analyte increases. In theory, using peak sum function to add multiple MRM transitions together can significantly improve sensitivity, but the assay specificity will occasionally be sacrificed due to the interference from the coeluted metabolites and/or other components from the biological matrix [12]. Coupling MS/MS with UPLC may improve both assay sensitivity and specificity by providing better chromatographic resolution and sharper peaks [14]. However, until now, challenges have existed for routinely analyzing oligonucleotides from biological matrices primarily due to the short life of UPLC columns.

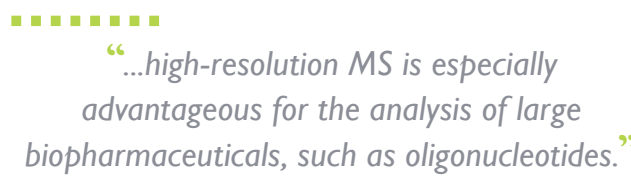

There is a growing interest in the application of full-scan high-resolution MS (HRMS) for the quantitative analysis of oligonucleotides $[15,16]$. In addition to quantitative information for the target oligonucleotides, HRMS can simultaneously provide additional information related to major metabolites without standard reference materials. The introduction of a nonhybrid, single-stage benchtop Orbitrap ${ }^{\mathrm{TM}}$ mass spectrometer with a quadrupole mass filter (Thermo Fisher Scientific) should make LC-HRMS more affordable/accessible for bioanalytical laboratories.

When using HRMS, compound-specific MS/MS tuning is not required for specificity; very high resolution $(R>10,000)$ in full-scan MS mode is sufficient. Because the fragmentation process intrinsically reduces the sensitivity of triple-quadrupole methods, HRMS in full-scan MS mode applications may theoretically improve the detection limits. HRMS instruments also have a much broader mass range compared with the triple-quadruple instruments. While a typical $m / z$ range is $80-1250$ for a Sciex API5000, an Exactive-based Orbitrap has a $\mathrm{m} / \mathrm{z}$ range of 200-4000. Therefore, HRMS is especially advantageous for the analysis of large biopharmaceuticals, such as oligonucleotides. On a benchtop Orbitrap with a quadrupole mass filter, a 1.0 $\mathrm{ng} / \mathrm{ml}$ LOQ was achievable for an 18-mer phosphorothioate DNA oligonucleotide with $10 \mu \mathrm{l}$ injection of the neat solution on a $3 \mu \mathrm{m} 2 \times 50$ Clarity ${ }^{\circledR}$-RP column, signaling potential applications, at least, in nonregulated environments [Cook K \& Bennett Pet al., Unpublished Data].

\section{Acknowledgements}

The author would like to thank Andrea Jones-Moore and James Fleming from LabCorp for critical review of the manuscript. The author also appreciates Kevin Cook and Patrick Bennett from ThermoScientific for providing relevant HRMS data and discussions.

\section{Financial \& competing interests disclosure}

The author has no relevant affiliations or financial involvement with any organization or entity with a financial interest in or financial conflict with the subject matter or materials discussed in the manuscript. This includes employment, consultancies, honoraria, stock ownership or options, expert testimony, grants or patents received or pending, or royalties. No writing assistance was utilized in the production of this manuscript.

\section{References}

1 Tremblay GA, Oldfield PR. Bioanalysis of siRNA and oligonucleotide therapeutics in biological fluids and tissues. Bioanalysis 1(3), 595-609 (2009).

2 Efler SM, Zhang L, Noll BO, Uhlmann E, Davis HL. Quantitation of oligodeoxynucleotides in human plasma with a novel hybridization assay offers greatly enhanced sensitivity over capillary electrophoresis. Oligonucleotides 15(2), 119-131 (2005).

3 Yu RZ, Baker B, Chappell A, Geary RS, Cheung E, Levin AA. Development of an ultrasensitive noncompetitive hybridization ligation enzyme-linked immunosorbent assay for the determination of phosphorothioate oligodeoxynucleotide in plasma. Anal. Biochem. 304(1), 19-25 (2002).
4 Shenk TE, Rhodes C, Rigby PW, Berg P. Biochemical method for mapping mutational alterations in DNA with S1 nuclease: the location of deletions and temperaturesensitive mutations in simian virus 40. Proc. Natl Acad. Sci. USA 72(3), 989-993 (1975).

5 Li ZJ, Li W, Dai G. Application of LC-MS for quantitative analysis and metabolite identification of therapeutic oligonucleotides. J. Pharmaceut. Biomed. Analy. 44 (2), 330-341 (2007). 
6 Deverre JR, Boutet V, Boquet D, Ezan E, Grassi J, Grognet JM. A competitive enzyme hybridization assay for plasma determination of phosphodiester and phosphorothioate antisense oligonucleotides. Nucleic Acids Res. 25(18), 3584-3589 (1997).

7 Wang L. Bioanalysis of oligonucleotide therapeutics: LC-MS/MS allows for accurate and sensitive HT quantification. Genet. Eng. Biotechn. News 31(2), 26-27 (2011).

8 Deng P, Chen X, Zhang G, Zhong D. Bioanalysis of an oligonucleotide and its metabolites by liquid chromatographytandem mass spectrometry. J. Pharm. Biomed. Anal. 52, 571-579 (2010).

9 Wang L, Yuan W, Zhao Y et al. Quantitation of TL0901 oligonucleotide in human plasma using LC-MS/MS. Presented at: AAPS Annual Meeting and Exposition. Los Angeles, CA, USA 8-12 November (2009).
10 Zhang G, Lin J, Srinivasan K, Kavetskaia O, Duncan JN. Strategies for bioanalysis of an oligonucleotide class macromolecule from rat plasma using liquid chromatography-tandem mass spectrometry. Anal. Chem. 79(9), 3416-3424 (2007).

11 Li Z, Schariter JA, Zhang J, Davis JC, Leone AM. Application of ultra-high performance liquid chromatography for chemical characterization of liposome-based therapeutic small-interference RNA. American Pharmaceutical Review 102-110 (2010).

12 Chen C, Wang L, Zhang Y et al. Quantitative determination of AS1411 oligonucleotide in monkey plasma using UPLC-PDA. Presented at: ISSX 15th Annual Meeting. San Diego, CA, USA 11-15 October (2009).

13 Tremblay G, Khalafaghian G, Legault J, Nielsen P, Bartlett A. Dual ligation hybridization assay for the specific determination of oligonucleotide therapeutics. Bioanalysis 3(5), 499-508 (2011).
14 Ivleva VB, Yu YQ, Gilar M. Ultraperformance liquid chromatography-tandem mass spectrometry (UPLC-MS/MS) and UPLC-MSE analysis of RNA oligonucleotides. Rapid Commun. Mass Spectrom. 24(17), 2631-2640 (2010).

15 Smith M. Characterization of a modified oligonucleotide together with its synthetic impurities using accurate mass measurements. Rapid Commun. Mass Spectrom. 25(4), 511-525 (2011).

16 Zou Y, Tiller P, Chen IW, Beverly M, Hochman J. Metabolite identification of small interfering RNA duplex by high-resolution accurate mass spectrometry. Rapid Commun. Mass Spectrom. 22(12), 1871-1881 (2008).

\section{- Patent}

101 Yu Z, Baker BF, Wu H. Nuclease-based method for detecting and quantifying oligonucleotides. Isis Pharmaceuticals Inc.: WO/2002/059137 (2003). 